\title{
Polarization Maintaining Mode Selective Coupler
}

\author{
Rand Ismaeel, Marcelo Botelho Alonso and Gilberto Brambilla \\ Optoelectronics Research Center, University Of Southampton, Southampton UK. \\ e-mail:rmni1g10@soton.ac.uk
}

\begin{abstract}
: fiber coupler composed of weakly fused few mode fiber and a single mode fiber is demonstrated. The coupler is capable of exciting specific higher order mode at the required spatial direction with high efficiency. Optimizing the length of the couplers together with a slow varying cross-section have shown to remove polarization and spatial degeneracy in the first and second high order mode.
\end{abstract}

OCIS codes: (060.0060) Fiber optics and optical communications; (060.1810) Buffers, couplers, routers, switches, and multiplexers.

\section{Introduction}

Higher order modes HOMs, have been recently suggested as an attractive solution for the predicted capacity crunch in communication networks [1]. Utilizing devices capable of propagating these HOMs is particularly continent for telecom systems for Mode-division multiplexing (MDM), where each of these mode have the potential to operate as an isolated channel. Allegiant configurations were demonstrated to generate these HOMs with high efficiency and purity, including photonic lanterns [2], asymmetric planar Y-junction splitters/combiners [3], and mode selective couplers $[4,5]$. The principle of mode selective couplers (MSC) is based on coupling the fundamental mode in the single mode fibre (SMF) to a single HOM in the few mode fibre (FMF) by phase matching the fundamental mode (FM) and the HOM in dissimilar diameter fibres. This work demonstrate experimental discrimination of spatially- or polarization- degenerate modes for weakly fused couplers. We prove the possibility of selectively excite one single spatial component of a $\mathrm{HOM}$ (the $L P_{11}$ and the $L P_{21}$ with a selected polarization.

\section{Weakly fused Mode Selective Couplers (WFC)}

In this geometry, the WFC is composed of two microfibers of different diameters, designed in a way that the propagation constants of the different modes in each MF are made equal.The modes guided my modal couplers show high polarization dependency. These HOMs with different polarizations or spatial field distribution, have a different effective index. Here the excitation of a selected spatially-non-degenerate $\mathrm{LP}_{11}$ and the $\mathrm{LP}_{21}$ mode in FMF-SMF WFC is demonstrated by increasing the difference between the effective indices of the two orthogonal modes which are degenerate in a cylindrically symmetric waveguide. The polarization discrimination capability of the coupler results from the coupler weakly fused cross section, which does not have a cylindrical symmetry and thus supports $\mathrm{LP}_{11(a)}$ and $\mathrm{LP}_{11(b)}$ (or $\mathrm{LP}_{21(a)}$ and $\mathrm{LP}_{21(b)}$ ) with different $\beta$. As the phase difference between modes is the product of effective index difference times length, in order to separate the two spatial component of the HOM which have distinct but close effective index, the uniform waist region of the coupler should have a long coupling length, enough to have a $\Delta \beta=\pi / 2$ between the two spatial modes at the end of the coupling region.

\section{Experiment}

To determine the polarization dependence/selectivity, two WFCs were manufactured for each of the $\mathrm{LP}_{11}$ and the $\mathrm{LP}_{21}$ mode. The first coupler (coupler 1) length were $L=2 \mathrm{~cm}$ while the length of coupler 2 was $L=5 \mathrm{~cm}$ in coupler 2. In all the couplers the SMF diameter was pre-tapered to the phase matching conditions given in [4] $(79 \mu \mathrm{m})$ for the $\mathrm{LP}_{11}$ and $(45 \mu \mathrm{m})$ for the $\mathrm{LP}_{21}$. The pre-tapered fiber was then aligned with the FMF and fused together using the modified flame brushing technique. The power transfer between both fibres was monitored in-situ using a power meter attached to both output ports, while light from a laser diode source was launched into a linear polarizer which was then connected to the SMF input port Fig. 1. The output of the FMF was monitored during the process and tapering was stopped when most of the power reached the coupled port.

The results shown in Fig. 2 show that in the case of the short couplers (Coupler1), it was possible to generate the $\mathrm{HOM}\left(\mathrm{LP}_{11}\right.$ or $\left.\mathrm{LP}_{21}\right)$ with high purity, However these modes were highly polarization dependent, where rotating the 


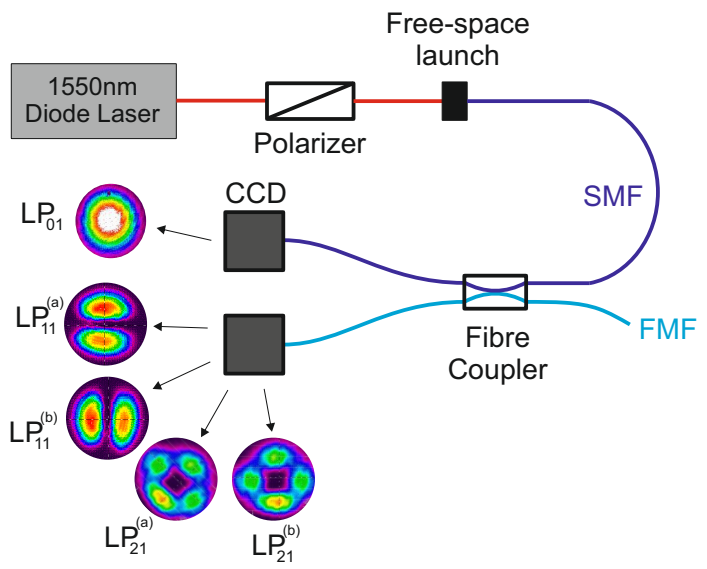

Fig. 1. Experimental set-up to monitor the spatial mode distribution in modal couplers.

polarization of the incident light by $90^{\circ}$ caused the mode spatial profile captured by the CCD camera to rotate by $90^{\circ}$. For the case of the coupler 2 , the situation was different,the same figure shows that rotating the polarizer has a minimum effect on the spatial field distribution. This seems to indicate that coupling occurs only with only one spatial mode. i.e. the spatial degeneracy has been removed.

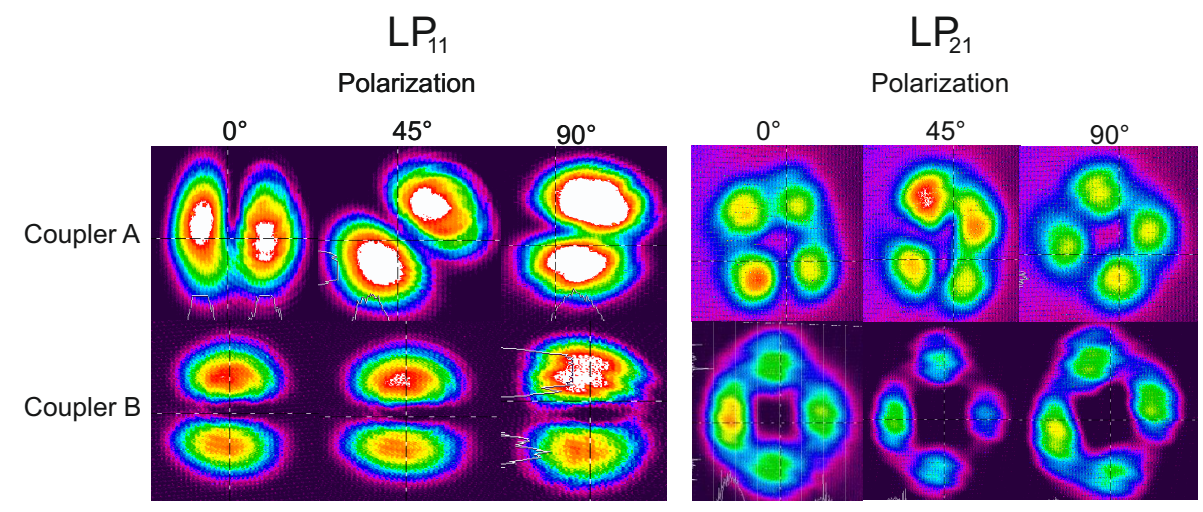

Fig. 2. Far field images of the $L P_{11}$ and $L P_{21}$ modes for coupler 1, coupler 2 at both polarization directions.

The different behavior of both (coupler 1) and long (coupler 2) couplers were noticed experimentally with high repeatability. Short couplers always exhibited mode orientation dependence on the polarization input, while long couplers can be unaffected by polarization azimuth change.

\section{References}

1. D. J. Richardson, J. M. Fini, and L. E. Nelson, "Space-division multiplexing in optical fibres," Nature Photonics 7, 354-362 (2013).

2. S. Leon-Saval, T. Birks, J. Bland-Hawthorn, and M. Englund, "Multimode fiber devices with single-mode performance," Opt. Lett. 30, 2545-2547 (2005).

3. J. D. Love, and N. Riesen. "Design of mode-sorting asymmetric Y-junctions." Applied optics 51, no. 15, 27782783 (2012).

4. R. Ismaeel, T. Lee, B. Oduro, Y. Jung, and G. Brambilla, "All-fiber fused directional coupler for highly efficient spatial mode conversion," Opt. Express 22, 11610-11619 (2014).

5. R. Ismaeel, G. Brambilla, "Removing the directional degeneracy of $L P_{11}$ mode in a fused-type mode selective coupler," in Lightwave Technology, Journal of , Accepted. doi: 10.1109/JLT.2016.2520209. 